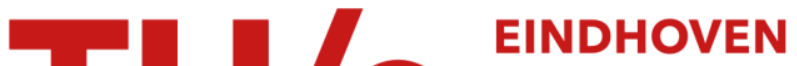 UNIVERSITY OF TECHNOLOGY
}

\section{Zero-shear stress relaxation and long time dynamics of a linear polyethylene melt: A test of Rouse theory}

Citation for published version (APA):

Padding, J. T., \& Briels, W. J. (2001). Zero-shear stress relaxation and long time dynamics of a linear polyethylene melt: A test of Rouse theory. Journal of Chemical Physics, 114(19), 8685-8693.

https://doi.org/10.1063/1.1368135

DOI:

$10.1063 / 1.1368135$

Document status and date:

Published: 01/01/2001

Document Version:

Publisher's PDF, also known as Version of Record (includes final page, issue and volume numbers)

Please check the document version of this publication:

- A submitted manuscript is the version of the article upon submission and before peer-review. There can be important differences between the submitted version and the official published version of record. People interested in the research are advised to contact the author for the final version of the publication, or visit the $\mathrm{DOI}$ to the publisher's website.

- The final author version and the galley proof are versions of the publication after peer review.

- The final published version features the final layout of the paper including the volume, issue and page numbers.

Link to publication

\section{General rights}

Copyright and moral rights for the publications made accessible in the public portal are retained by the authors and/or other copyright owners and it is a condition of accessing publications that users recognise and abide by the legal requirements associated with these rights.

- Users may download and print one copy of any publication from the public portal for the purpose of private study or research.

- You may not further distribute the material or use it for any profit-making activity or commercial gain

- You may freely distribute the URL identifying the publication in the public portal.

If the publication is distributed under the terms of Article $25 \mathrm{fa}$ of the Dutch Copyright Act, indicated by the "Taverne" license above, please follow below link for the End User Agreement:

www.tue.nl/taverne

Take down policy

If you believe that this document breaches copyright please contact us at:

openaccess@tue.nl

providing details and we will investigate your claim. 


\section{AIP Chemicital Prysics}

\section{Zero-shear stress relaxation and long time dynamics of a linear polyethylene melt: A test of Rouse theory}

J. T. Padding and W. J. Briels

Citation: J. Chem. Phys. 114, 8685 (2001); doi: 10.1063/1.1368135

View online: http://dx.doi.org/10.1063/1.1368135

View Table of Contents: http://jcp.aip.org/resource/1/JCPSA6/v114/i19

Published by the American Institute of Physics.

\section{Related Articles}

Effects of alignment layer thickness on the pretilt angle of liquid crystals APL: Org. Electron. Photonics 3, 270 (2010)

Effects of alignment layer thickness on the pretilt angle of liquid crystals Appl. Phys. Lett. 97, 243306 (2010)

Field-theoretic model of inhomogeneous supramolecular polymer networks and gels J. Chem. Phys. 133, 174903 (2010)

Origin of translocation barriers for polyelectrolyte chains JCP: BioChem. Phys. 3, 11 B610 (2009)

Origin of translocation barriers for polyelectrolyte chains J. Chem. Phys. 131, 194903 (2009)

\section{Additional information on J. Chem. Phys.}

Journal Homepage: http://jcp.aip.org/

Journal Information: http://jcp.aip.org/about/about_the_journal

Top downloads: http://jcp.aip.org/features/most_downloaded

Information for Authors: http://jcp.aip.org/authors

\section{ADVERTISEMENT}

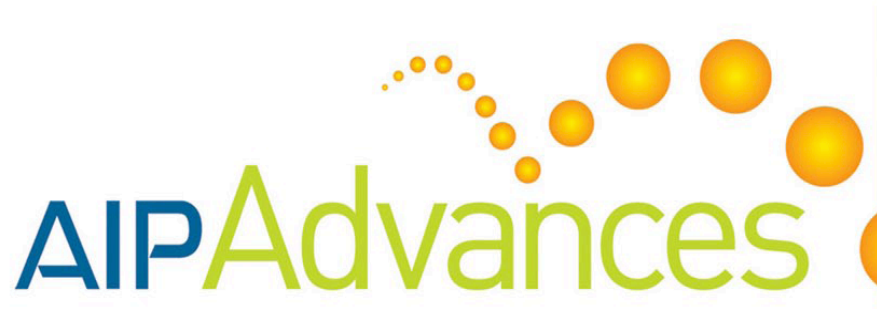

Submit Now

Explore AIP's new

open-access journal

Article-level metrics now available

Join the conversation!

Rate \& comment on articles 


\title{
Zero-shear stress relaxation and long time dynamics of a linear polyethylene melt: A test of Rouse theory
}

\author{
J. T. Padding ${ }^{\text {a) }}$ and W. J. Briels ${ }^{\text {b) }}$ \\ Computational Dispersion Rheology, Department of Applied Physics, University of Twente, P.O. Box 217, \\ 7500 AE Enschede, The Netherlands
}

(Received 17 October 2000; accepted 6 March 2001)

\begin{abstract}
Results of united atom molecular dynamics simulations of a $n-\mathrm{C}_{120} \mathrm{H}_{242}$ melt at $450 \mathrm{~K}$ are presented. It is shown that the results of mean square displacement, dynamic structure factor, end-to-end vector autocorrelation, and shear relaxation modulus can consistently be described by the Rouse model with a single set of fit parameters, provided the length scales involved are larger than the statistical segment length $b \approx 1.2 \mathrm{~nm}$. On smaller length scales the stiffness of the chain becomes prominent, and the results deviate increasingly from the Rouse predictions. The shear relaxation modulus $G(t)$ is determined from the stress autocorrelation function from both atomic and molecular points of view. The integrals $\int G(t) d t$ are found to be identical after 1 ps and a Rouse description is shown to coincide for time scales larger than $0.4 \mathrm{~ns}$. Compared to experimental values, the measured diffusion coefficient is overestimated by $63 \%$ and the viscosity is underestimated by $38 \%$, consistent with molecular dynamics simulations of small molecules. (c) 2001 American Institute of Physics.
\end{abstract}

[DOI: $10.1063 / 1.1368135]$

\section{INTRODUCTION}

A melt of polyethylene (PE) can be viewed as a prototype of a large spectrum of different polymer species, which all have the common feature that they are long and linear. Both its simple chemical structure and its technological relevance have made PE the favorite subject of modeling and simulation efforts on computers. However, the level of sophistication and atomic detail is always limited by the computational cost which can become quite enormous because of the large time and length scales involved in the dynamic properties of polymers. For instance, atomic simulations of melts of very long (thousands of monomers) chains are still out of reach of current-day computer power. Coarse grained models have been applied to overcome this problem and some are quite successful in reproducing experimentally observed scaling laws of polymer melt properties, such as the diffusion coefficient and viscosity. However, quantitative agreement with experiment is usually better when atomic simulations are applied. Therefore the results of atomic simulations are important to check the validity of coarse grained models and to calculate the parameters occurring in these models.

In this paper we report diffusion, dynamic structure factor, zero-shear relaxation modulus, and viscosity results of atomic molecular dynamics (MD) simulations of a melt of $n-\mathrm{C}_{120} \mathrm{H}_{242}$ chains. Several other studies have been reported on simulations of melts of polymers of comparable size. ${ }^{1-9}$ They all suggest that individual properties of these melts can well be described by the Rouse model. ${ }^{10}$ In this paper we will test whether the Rouse model can be used to predict the time correlation functions of a comprehensive set of physical

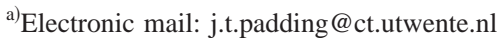

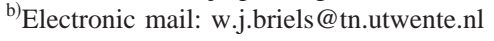

processes by using one single set of parameters. At the same time our results provide a benchmark for a coarse grained modeling of polyethylene (to be published).

Paul and coworkers have performed molecular dynamics simulations of a melt of $n-\mathrm{C}_{100} \mathrm{H}_{202}$ chains. ${ }^{1,2}$ They found that translational and rotational diffusion of the chains can consistently be described by the Rouse model, but that systematic deviations show up in the internal dynamics of the chains. Also, subdiffusive behavior was observed in the chain dynamics, not predicted by the Rouse model. Similar results were found by Harmandaris et al. for chains in polydisperse blends. ${ }^{3}$ Mondello and coworkers investigated a number of chain lengths between $\mathrm{C}_{10}$ and $\mathrm{C}_{66}{ }^{5,6}$ By use of the Green-Kubo (GK) relation they were able to calculate the zero-shear viscosity $\eta_{0}$ for chains up to $\mathrm{C}_{16}$ by integrating the zero-shear stress relaxation curves obtained from equilibrium molecular dynamics simulations (EMD). They were not able to determine the viscosity by this method for chains longer than $\mathrm{C}_{16}$. The reason for this, as was already shown by Cui and coworkers for the case of liquid decane, ${ }^{11}$ is that the integration time must be at least as long as the longest relaxation time, which increases enormously with chain length. A more direct method to estimate the viscosity is provided by the nonequilibrium molecular dynamics (NEMD) technique in which a shear flow is imposed on the system. By measuring relevant components of the stress tensor, the viscosity may be determined as a function of shear rate. Using this method, Mondello et al. showed that $\eta_{0}$ of medium long chains may be estimated from the longest relaxation time measured in EMD simulations by invoking the Rouse model. ${ }^{5,6}$ They found that the estimates are in good agreement with extrapolated NEMD results (within 20\%). Moore et al. have performed NEMD simulations of a $\mathrm{C}_{100}$ melt (close to our $\mathrm{C}_{120}$ ) at various shear rates. ${ }^{7}$ They ob- 
served substantial shear thinning at shear rates larger than the inverse rotational diffusion time. Unfortunately, they were unable to observe a Newtonian plateau and experienced problems in extrapolating to zero shear. This is common to NEMD simulations in which large shear rates are needed to obtain significant results.

The above results seem to indicate that many properties of medium long chains may well be described by invoking the Rouse model. This is quite remarkable since the Rouse model assumes that the surrounding chains merely constitute a stochastic background to a chain of harmonically bound beads without excluded volume, and thus is not a very realistic model. It is therefore not at all obvious that a chain which behaves Rouse-like with respect to one property should also behave Rouse-like with respect to another property. It is our aim with this paper to investigate whether one set of Rouse parameters exists which describes all dynamic properties of a $\mathrm{C}_{120}$ melt. In particular we want to include the full zero-shear stress relaxation modulus among the data to be described, and not just the time integrated modulus, i.e., $\eta_{0}$.

After we completed this work, Harmandaris et al. published a study which is quite similar in spirit. ${ }^{9}$ Using NEMD simulations, and EMD simulations in the case of a $\mathrm{C}_{24}$ melt, they showed that all dynamic processes of $\mathrm{C}_{24}$ and $\mathrm{C}_{78}$ melts, including shear relaxation processes up to $3 \mathrm{~ns}$, may well be described by the Rouse model. In this paper we will supply additional evidence and also show that a $\mathrm{C}_{120}$ melt may be described by the Rouse model, at least for processes lasting up to $1 \mathrm{~ns}$. Since all our calculations will be based on EMD simulations, the linear response regime will be guaranteed.

This paper is organized as follows. In Sec. II we summarize the simulation model and the methodology used to obtain a well-equilibrated melt. The method of calculation of the various correlation functions and transport properties is summarized in Sec. III. The results are presented and discussed in Sec. IV. We summarize our conclusions in Sec. V. In the Appendix we present the Rouse predictions for the measured quantities.

\section{SIMULATION MODEL AND METHODOLOGY}

MD simulations were performed on four independent boxes of amorphous PE using the GROMOs package. ${ }^{12}$ Each box consisted of 12 chains of 120 monomer units $\left[-\mathrm{CH}_{2}-\right]$, a system small enough to make calculations computationally feasible, yet large enough to avoid significant interactions of a chain with its periodic images. Moreover, the $\mathrm{CH}_{2}$ and $\mathrm{CH}_{3}$ groups are modeled as united atoms (UA) in order to reduce the number of atoms in the actual simulation. Studies by Paul and coworkers have shown the ability of UA models to yield the same dynamic results as more expensive explicit atom models. ${ }^{1,13}$ The simulated systems were all subject to cubic periodic boundary conditions. Bond vibrations and bond angle vibrations were treated by harmonic potentials

$$
V(b)=\frac{1}{2} k_{b}\left(b-b_{0}\right)^{2}
$$

for all bonds, and

$$
V(\theta)=\frac{1}{2} k_{\theta}\left(\theta-\theta_{0}\right)^{2}
$$

TABLE I. Force field parameters. ${ }^{a}$

\begin{tabular}{lcl}
\hline \hline \multicolumn{1}{c}{ Parameter } & Value & \multicolumn{1}{c}{ Units } \\
\hline$k_{b}$ (bond) & 3.3475 & $10^{5} \mathrm{~kJ} \mathrm{~mol}^{-1} \mathrm{~nm}^{-2}$ \\
$b_{0}$ & 0.153 & $\mathrm{~nm}$ \\
$k_{\theta}$ (bending) & 519.6 & $\mathrm{~kJ} \mathrm{~mol}^{-1} \mathrm{rad}^{-2}$ \\
$\theta_{0}$ & 114.0 & $\mathrm{deg}$ \\
$c_{0}$ (torsion) & 9.2789 & $\mathrm{~kJ} \mathrm{~mol}^{-1}$ \\
$c_{1}$ & 12.156 & \\
$c_{2}$ & -13.120 & \\
$c_{3}$ & -3.0597 & \\
$c_{4}$ & 26.24 & \\
$c_{5}$ & -31.495 & $\mathrm{~kJ} \mathrm{~mol}$ \\
$\epsilon\left(\mathrm{CH}_{2}\right)$ & 0.3908 & \\
$\epsilon\left(\mathrm{CH}_{3}\right)$ & 0.9480 & $\mathrm{~nm}$ \\
$\sigma\left(\mathrm{CH}_{2}\right)$ & 0.3930 & \\
$\sigma\left(\mathrm{CH}_{3}\right)$ & 0.3930 & \\
\hline
\end{tabular}

${ }^{\mathrm{a}}$ Torsion parameters taken from Ryckaert and Bellemans (Ref. 14). Other parameters taken from Smit, Karaborni, and Siepmann (Ref. 15).

for all bond angles, where $b_{0}$ and $\theta_{0}$ are the equilibrium bond length and angle. The force constants for bond and bond angle vibrations are $k_{b}$ and $k_{\theta}$, respectively. The dihedral rotations were described with the rotational potential of Ryckaert and Bellemans, ${ }^{14}$

$$
V(\varphi)=\sum_{n=0}^{5} c_{n} \cos ^{n}(\varphi) .
$$

The intermolecular interactions and the interactions between atoms separated by four or more atoms in the same molecule were described by Lennard-Jones potentials

$$
V(r)=4 \epsilon\left[(\sigma / r)^{12}-(\sigma / r)^{6}\right] .
$$

Parameters are listed in Table I. We used a $1.15 \mathrm{~nm}$ cutoff for the Lennard-Jones interactions. The equations of motion were solved using the leapfrog algorithm with a conservative time step of 1 fs. Simulations of the NVT ensemble were performed using a weak coupling scheme to a temperature bath of $450 \mathrm{~K}$ with a coupling constant of $0.1 \mathrm{ps} .{ }^{16}$ The density was set to $0.7614 \mathrm{~g} / \mathrm{cm}^{3}$, close to the normal pressure density at $450 \mathrm{~K} .{ }^{17}$ The average pressure from the simulation was $p \approx 54$ atm.

For a correct sampling of the pressure tensor autocorrelation it is important to have a fully equilibrated melt in which there is no average stress present. This is far from trivial for complex fluids like polyethylene. A number of ways have been suggested by different authors. Forrest and Suter have time-coarse grained the atomic interactions to improve the sampling efficiency. ${ }^{18}$ Tschöp et al. suggested a spatial-coarse graining for polycarbonates, after which the chemical details are reintroduced into the coarse grained chains. ${ }^{19}$ Quite successful in obtaining a well-equilibrated melt is the end-bridging Monte Carlo algorithm, as suggested by Pant and Theodorou, which, however, yields polydisperse melts because of the connectivity-altering moves. ${ }^{20,3}$ Van der Vegt et al. have investigated the influence of three different ways of generating initial configurations on the solubilities of small molecules in amorphous polymer melts. ${ }^{21}$ In one of the methods a dilute system is slowly compressed, during which process only the repulsive parts of the nonbonded 
Lennard-Jones potentials are taken into account. The configurations of this paper have been prepared this way. After reintroduction of the full potential, the melt was equilibrated at the final density for $10 \mathrm{~ns}$. The average stress was measured during several simulations of $1 \mathrm{~ns}$ each and found to be small enough to have no influence on the stress-stress correlation functions described in Sec. IV.

The production time was $35 \mathrm{~ns}$, the viscosity measurements were done in a subsequent $10 \mathrm{~ns}$ run to ensure maximum relaxed systems.

\section{CALCULATION OF CORRELATION FUNCTIONS AND TRANSPORT PROPERTIES}

We have measured three time-dependent mean square displacements $g_{\mathrm{at}}(t), g_{\mathrm{bl}}(t)$, and $g_{\mathrm{cm}}(t)$, which are defined as follows:

$$
\begin{aligned}
& g_{\mathrm{at}}(t)=\frac{1}{N} \sum_{i=1}^{N}\left\langle\left[\mathbf{r}_{i}(t)-\mathbf{r}_{i}(0)\right]^{2}\right\rangle, \\
& g_{\mathrm{bl}}(t)=\frac{1}{N / 20} \sum_{j=1}^{N / 20}\left\langle\left[\mathbf{r}_{j}^{\mathrm{bl}}(t)-\mathbf{r}_{j}^{\mathrm{bl}}(0)\right]^{2}\right\rangle, \\
& g_{\mathrm{cm}}(t)=\left\langle\left[\mathbf{r}^{\mathrm{cm}}(t)-\mathbf{r}^{\mathrm{cm}}(0)\right]^{2}\right\rangle,
\end{aligned}
$$

where $\mathbf{r}_{i}$ is the position of the $i$ th carbon atom, $\mathbf{r}_{j}^{\mathrm{bl}}$ is the position of the $j$ th "blob," as we will explain next, and $\mathbf{r}^{\mathrm{cm}}$ is the center of mass of the chain. In a following paper we will present mean square displacement results of a coarse grained model of polyethylene, in which the smallest particles are blobs. Each blob is supposed to mimic the behavior of the center of mass of a collection of 20 successive carbon atoms. To validate this coarse grained model, we measure $g_{\mathrm{bl}}$ in the current atomic simulation. The diffusion coefficient is calculated from the mean square displacement by

$$
D=\lim _{t \rightarrow \infty} \frac{g_{\mathrm{cm}}(t)}{6 t} .
$$

The coherent dynamic structure factor of a single chain can be measured by means of neutron spin echo spectroscopy. It is defined as

$$
S(q, t)=\frac{1}{N} \sum_{i=1}^{N} \sum_{j=1}^{N}\left\langle\exp \left\{i \mathbf{q} \cdot\left[\mathbf{r}_{i}(t)-\mathbf{r}_{j}(0)\right]\right\}\right\rangle,
$$

where the two summations run over the (united) atoms of one chain.

The atomic (molecular) stress tensor is defined as

$$
\boldsymbol{\sigma}=\frac{1}{V}\left[\sum_{i=1}^{N} m_{i} \mathbf{v}_{i} \mathbf{v}_{i}+\sum_{i>j}\left(\mathbf{r}_{i}-\mathbf{r}_{j}\right) \mathbf{F}_{i j}\right],
$$

where $\mathbf{v}$ is the atomic (molecular) velocity, $\mathbf{r}$ is the atomic (molecular center of mass) position, and $\mathbf{F}_{i j}$ is the force exerted on atom (molecular center of mass) $i$ by atom (molecule) $j$. The molecular stress tensor contains an antisymmetric part because the total force on a molecule exerted by another molecule is not directed along the line of centers of the molecules, producing a torque on the molecules. ${ }^{22}$ For calculating the shear viscosity we need the symmetric part.

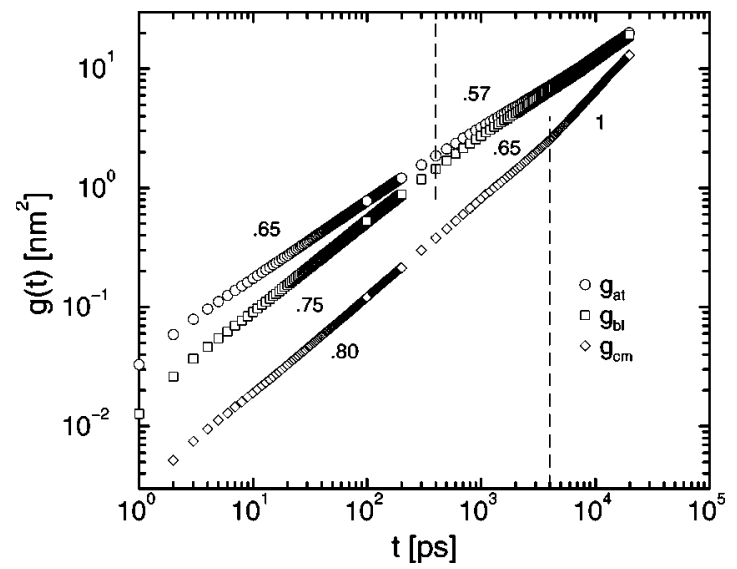

FIG. 1. Mean square displacement of the atom (circles), blob (squares), and center of mass (diamonds) positions as a function of time on a double logarithmic scale. The numbers are the estimated power law exponents $x$. The dashed lines indicate the transition times between the different power law regimes.

Daivis and Evans ${ }^{23}$ have shown that the zero shear relaxation modulus is related to the symmetrized traceless part $\mathbf{P}$ of the stress tensor $\boldsymbol{\sigma}$ by

$$
G(t)=\frac{V}{10 k_{B} T}\langle\mathbf{P}(t): \mathbf{P}(0)\rangle .
$$

The double contraction means in practice that we average over five independent contributions: $P_{x y}, P_{x z}, P_{y z}, 1 / 2\left(P_{x x}\right.$ $\left.-P_{y y}\right)$, and $1 / 2\left(P_{x x}-P_{z z}\right)$ [we get no additional information from $1 / 2\left(P_{y y}-P_{z z}\right)$ since $\mathbf{P}$ is traceless]. The viscosity is given by the infinite time integral of Eq. (11). It has been proved by Allen that viscosities calculated from the atomic and molecular approach are equal. ${ }^{24}$ Several simulation studies have shown this to be correct. ${ }^{5,11}$ The difference between the viscosity calculated from the molecular tensor and that from the atomic tensor is found to converge to zero much faster than the longest relaxation time of the molecule. This was confirmed in test runs of our system in which we calculated the shear relaxation modulus from both atomic and molecular stress tensors. However, the behavior of the atomic stress autocorrelation is dominated by the strong oscillatory behavior due to the bond stretching vibrations. Thus for practical purposes, the molecular tensor formalism is more efficient since it allows the use of a relatively large sampling time interval. We have chosen to measure the molecular pressure tensor every $50 \mathrm{fs}$. To make certain that we perform an accurate integration of the data, we have also calculated the integral with a 5 fs interval for the shorter time scales. The two integrals were found to be identical.

\section{RESULTS AND DISCUSSION}

In this section we will analyze the large time dynamics of the PE melt. We will explore the Rouse model and its boundaries of applicability to real PE chains.

\section{A. Mean square displacement}

In Fig. 1 we present the time-dependent mean square displacement results from the simulations. The results have been averaged over four independent boxes, allowing us to 


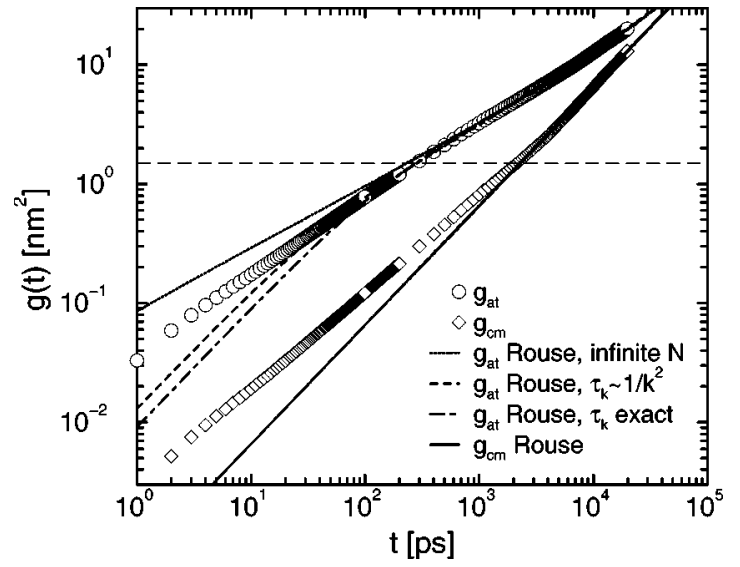

FIG. 2. Mean square displacement of the atom (circles) and center of mass (diamonds) positions. The lines are fits to the Rouse model. The dotted line is for an infinite Rouse chain $\left(N \rightarrow \infty, \tau_{1}=6.5 \mathrm{~ns}\right)$, the dashed line for a finite Rouse chain, with the approximation $\tau_{k} \propto 1 / k^{2}\left(N=20, \tau_{1}=7.0 \mathrm{~ns}\right)$, and the dot-dashed line for a finite Rouse chain with the exact expression for $\tau_{k}$ $\left(N=14, \tau_{1}=6.5 \mathrm{~ns}\right)$, see Eq. (A4). The statistical segment length $b^{2}$ $=1.49 \mathrm{~nm}$ is indicated by the long dashed horizontal line. Above this line the Rouse predictions are correct.

estimate the errors. The shortest time behavior $(t \leqslant 1 \mathrm{ps})$ is ballistic and not shown in the graph. After the ballistic regime, we observe two power law regimes $g(t) \propto t^{x}$ for $g$ at and $g_{b l}$. The atomic diffusion results reveal an exponent $x$ $=0.65 \mathrm{p}$ to $200 \mathrm{ps}$, after which an exponent $x=0.57$ sets in. The initial value compares well with the results of molecular dynamics simulations of $\mathrm{C}_{100} \mathrm{H}_{202}$ melts by Paul, Smith, and Yoon. ${ }^{1}$ They measured the mean square displacements of central and end monomers of each chain separately, which on average yields an exponent of $x=0.67$. For the blob diffusion results we measure an exponent $x=0.75$ up to $400 \mathrm{ps,}$ after which an exponent $x=0.65$ sets in. These higher exponents should be expected, since in going to the blob level we average over some of the atomic movement (in the ultimate limit of a chain being represented by one blob, $g_{\text {bl }}$ and $g_{\mathrm{cm}}$ would be the same). For both atomic and blob mean square displacements we expect free diffusion at larger time scales. The chain center of mass diffusion $g_{\mathrm{cm}}$ does indeed display free diffusive behavior after $t=4 \mathrm{~ns}$. Before this time, subdiffusive behavior $(x=0.80)$ is observed. The subdiffusive exponent compares well to both simulation ${ }^{1}$ and neutron spin echo spectroscopy ${ }^{2}$ results of a $\mathrm{C}_{100} \mathrm{H}_{202}$ melt $(x=0.83)$.

Now we want to investigate how closely the observed mean square displacement results resemble Rouse behavior. In the Rouse model, segmental mean square displacements show a subdiffusive regime $g_{\text {at }}(t) \propto t^{1 / 2}$ before crossing over to free diffusion. However, this is only true in the limit of $N$ going to infinity. For finite $N$ there is a transition from an early $t^{1}$ regime to a sublinear exponent. This transition occurs at the fastest relaxation time in the Rouse chain, which is $\tau_{N-1}$ in Eq. (A4). In Fig. 2 we present the result of fitting the three independent parameters of the Rouse model to the observed atomic and chain diffusion data. The three fit parameters are the diffusion coefficient of the chain $D$, the Rouse time $\tau_{1}$, and the number of statistical segments $N$. In the Rouse model, the center of mass of a chain will always diffuse freely, so a fit to the long time behavior of $g_{\mathrm{cm}}$ will uniquely determine $D$. We find a self-diffusion coefficient of $D=(1.09 \pm 0.09) \times 10^{-6} \mathrm{~cm}^{2} / \mathrm{s}$, which is in excellent agreement with the observations of Harmandaris and coworkers who found $D=(1.16 \pm 0.2) \times 10^{-6} \mathrm{~cm}^{2} / \mathrm{s}$ for a $\mathrm{C}_{117}$ chain, and $D=(1.0 \pm 0.1) \times 10^{-6} \mathrm{~cm}^{2} / \mathrm{s}$ for a $\mathrm{C}_{128}$ chain at the same temperature and approximately the same density as our simulation. ${ }^{3}$ Pearson et al. ${ }^{17}$ have measured self-diffusion coefficients by means of field gradient NMR at $450 \mathrm{~K}$ experimentally for a large range of molecular weights, although not at the weight of $\mathrm{C}_{120}$ chains. An interpolation of their data yields $D_{\text {exp }} \approx 0.67 \times 10^{-6} \mathrm{~cm}^{2} / \mathrm{s}$. The discrepancy is not specific to our united atom model. It is well-known that diffusion coefficients from MD simulations tend to overestimate the self-diffusion coefficient, especially at high packing fractions. For a systematic study the reader is referred to the work of Dysthe and coworkers. $^{25}$

From the diffusion coefficient we can calculate the segmental friction coefficient $\zeta$. However, $\zeta$ still depends on the size of the statistical segment. One can easily show this by writing $\zeta=m \xi$, in which $m$ is the mass of the statistical segment and $\xi$ is the friction "frequency.' If we apply this to Eq. (A7) we find $D=k_{B} T /(N m \xi)=k_{B} T /(M \xi)$, where $M$ is the total mass of the chain. Since within the Rouse model the diffusion coefficient of a given chain must not depend on how many segments we choose to divide it in, $\xi$ must be a property of the chain independent of our choice of statistical segment length (but still dependent on other factors, such as temperature). We find $\xi=(19.0 \pm 1.6) \mathrm{ps}^{-1}$ for the friction frequency. Harmandaris et al. have calculated the friction coefficient of a $\mathrm{CH}_{2}$ monomer, which is then shown to be independent of the chain length for chains longer than $\mathrm{C}_{70}$. If we calculate the monomeric friction coefficient we find $\zeta$ $=(4.45 \pm 0.37) \times 10^{-13} \mathrm{~kg} / \mathrm{s}$, in accordance with their results. ${ }^{3}$ However, we remind the reader that the number of segments $N$ in the Rouse model should not be set equal to the number of monomers in the chain, since one monomer does not make a statistical segment, as we will show later on.

Now we consider the atomic mean square displacements, shown in Fig. 2. The value of the sublinear exponent in the atomic mean square displacement depends on both the Rouse time $\tau_{1}$ and the number of statistical segments $N$. It was found that $N$ has little influence on the second regime of $g_{\text {at }}(t$ ) (for not too small $N$ ), so we used the regime from 1 to 20 ns to fit the Rouse time. Finally we varied $N$ to obtain the best fit for times below $1 \mathrm{~ns}$. Applying the exact expression for the relaxation time spectrum, Eq. (A4), we obtained $\tau_{1}$ $=(6.5 \pm 0.3) \mathrm{ns}$ and $N=14 \pm 2$. Applying the approximation $\tau_{k} \propto 1 / k^{2}$ [Eq. (A5)], we obtained slightly different parameters, $\tau_{1}=(7.0 \pm 0.3) \mathrm{ns}$ and $N=20 \pm 3$. Since $N$ is found to be relatively low, this approximation fails at short times, the first set of parameters is the more correct one. For comparison we have also included the results of a fit with an infinite Rouse chain $(N \rightarrow \infty)$, in which case $\tau_{1}=6.5 \mathrm{~ns}$ and a $t^{1 / 2}$ regime occurs. Clearly, the deviation of the measured exponent $x=0.57$ from the ideal value of 0.5 is due to the small number of statistical segments. From this study we can conclude that a statistical segment of polyethylene at $450 \mathrm{~K}$ is comprised of about $120 / 14=8.6$ monomers. The size of 


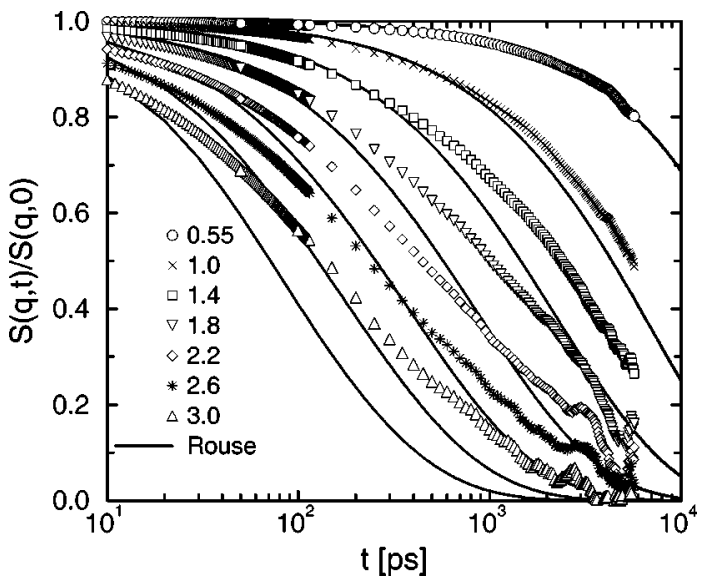

FIG. 3. Dynamic structure factor for seven different magnitudes of the wave vector. The symbols are the simulation data. The full curves are the Rouse fits, Eq. (A9).

this segment, $b^{2}$, is given by the combination of Eqs. (A7) and (A4),

$$
b^{2}=12 N D \tau_{1} \sin ^{2}\left(\frac{\pi}{2 N}\right)
$$

which yields $b^{2}=1.49 \mathrm{~nm}^{2}$. (If we would have used $N=20$ and $\tau_{1}=7.0 \mathrm{~ns}$ we would have found $b^{2}=1.13 \mathrm{~nm}^{2}$ for a segment comprised of six monomers.) It is apparent from Fig. 2 that it is this length (rather than a characteristic time) which determines when Rouse theory sets in. When both $g_{\text {at }}$ and $g_{\mathrm{cm}}$ have moved more than $b^{2}$, the Rouse theory predictions coincide with the molecular dynamics results. Consequently, the atomic results coincide much earlier than the chain center of mass results (after 0.4 and 4 ns, respectively). Below these limits the Rouse model fails because of the neglect of molecular stiffness, as was pointed out by Harnau and coworkers. ${ }^{26}$ Other factors may be important as well. For instance, Richter et al. suggested that an extra (internal) friction term may be necessary to explain experimental results. ${ }^{27}$ This has, however, been questioned by Harnau et al. ${ }^{28,29}$ In this paper we will not elaborate on this matter. Whenever "chain stiffness", is mentioned, the possibility of these other effects should be kept in mind as well.

\section{B. Dynamic structure factor}

The dynamic structure factor is experimentally the best accessible quantity to check the validity of the Rouse model. The neutron spin echo spectroscopy experiments described in Ref. 2 already formed a critical test of the Rouse model, and our conclusions correspond with those of the authors. In Fig. 3 we present the normalized dynamic structure factors for seven wave vectors ranging from $q=0.55$ to $3.0 \mathrm{~nm}^{-1}$ together with the Rouse predictions, Eq. (A9). The fit parameters were determined as follows: for $q \ll 2 \pi / R$, where $R$ is the end-to-end vector, the wave vector is so small that it only probes the overall diffusion of the chain. Thus we have used the first wave vector to determine $D$, independent of the mean square displacement measurements. We obtained $D$ $=(1.15 \pm 0.08) \times 10^{-6} \mathrm{~cm}^{2} / \mathrm{s}$, which is in prefect agreement with the previous results. The Rouse time $\tau_{1}$ and the number

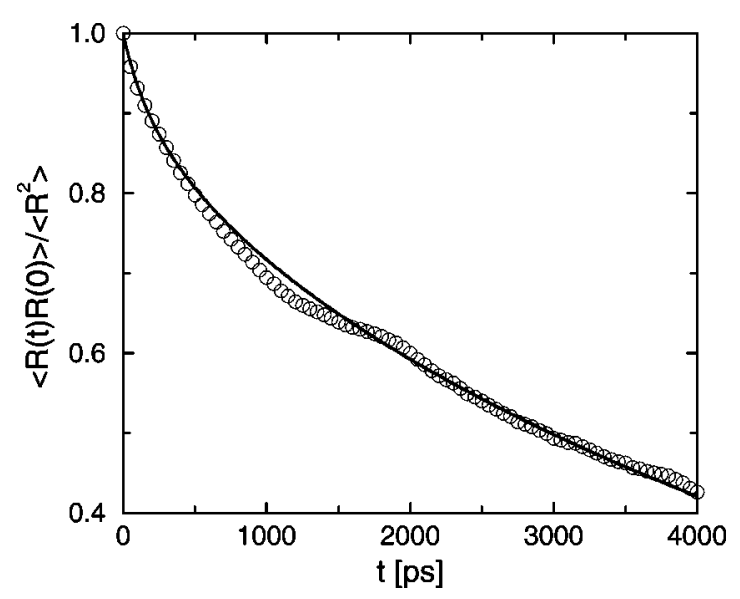

FIG. 4. Orientational autocorrelation function of the end-to-end vector. The circles are the simulation data, the full curve is the Rouse fit, Eq. (A11).

of segments $N$ are of importance for the larger wave vectors. A combined fit yields $\tau_{1}=(6.5 \pm 0.5) \mathrm{ns}$ and $N=15 \pm 5$. The large uncertainty in $N$ stems from the fact that the smallest wave vectors are relatively insensitive to $N$, while the larger wave vectors cannot be described correctly by any $N$ whatsoever. For $q \geqslant 1.4 \mathrm{~nm}^{-1}$ the Rouse curves decay too fast compared with the simulation data, leading to underestimated structure factors for large times (but an overestimate for times lower than $\sim 50 \mathrm{ps}$ ). This value of $q$ corresponds to a half-wavelength of $\pi / q=2.24 \mathrm{~nm}$, which is in the same order of magnitude as the statistical segment length $b$. Again, we conclude that the Rouse model is capable of correctly describing the behavior of a polymer chain, but only on length scales larger than the statistical segment length. Below this length scale the stiffness of the chain becomes important, leading to a slower decay of the dynamic structure factor.

\section{End-to-end vector}

The end-to-end vector measures the position of the last segment with respect to the first segment of a chain, $\mathbf{R}=\mathbf{r}_{N}$ $-\mathbf{r}_{1}$. For a Gaussian chain, such as the Rouse chain, the average squared magnitude of the end-to-end vector is given by Eq. (A10), which yields $20.9 \mathrm{~nm}^{2}$ if we use $N=14$ and $b^{2}=1.49 \mathrm{~nm}^{2}$, and $22.6 \mathrm{~nm}^{2}$ if we use $N=20$ and $b^{2}$ $=1.13 \mathrm{~nm}^{2}$. In the simulations we measured $\left\langle R^{2}\right\rangle=(23.8$ $\pm 4.0) \mathrm{nm}^{2}$, which is in rather good agreement with the Rouse prediction if we take into account that the end-to-end vector is measured from the first to the 120th carbon atom in the atomic case and between the centers of the first and last statistical segments in the case of a Rouse chain.

The dynamic quantity we are interested in is the orientational autocorrelation function for the end-to-end vector $\langle\mathbf{R}(t) \cdot \mathbf{R}(0)\rangle /\left\langle R^{2}\right\rangle$. It measures the rate at which the chain becomes uncorrelated with itself by means of rotational diffusion. Figure 4 presents the decay of the normalized end-toend vector autocorrelation function from the simulations including a fit with the Rouse prediction of Eq. (A11). One can vary both the number of statistical segments $N$ and the Rouse time $\tau_{1}$ to obtain a best fit. We have used the limiting single exponential behavior to determine the Rouse time and ad- 


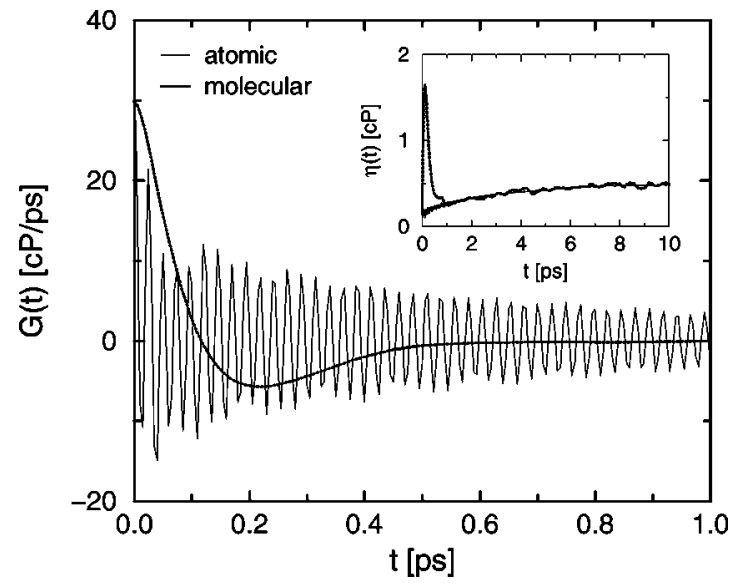

FIG. 5. Comparison of the short-time behavior of the shear relaxation modulus determined from the atomic (line) and molecular (dotted line) stress tensor. The inset shows the integral up to time $t$. After 1 ps the atomic and molecular integrals become identical.

justed $N$ to get the best fit for short time behavior. This procedure yields $\tau_{1}=(6.0 \pm 0.5) \mathrm{ns}$ and $N=13 \pm 3$, in agreement with the fit parameters obtained from the mean square displacement data. The fit can be made fairly accurate because the end-to-end vector is a property of the entire chain and much larger than the statistical segment length $b$.

\section{Shear relaxation modulus and viscosity}

The shear relaxation modulus $G(t)$ is calculated from the autocorrelation of the stress tensor, Eq. (11). As explained in Sec. III the stress tensor can be determined from an atomic or a molecular point of view. In Fig. 5 both results are presented for a sampling interval of $5 \mathrm{fs}$. The atomic shear relaxation modulus shows strong oscillatory behavior due to fast vibrations of the bonds in the chain, while the molecular shear relaxation modulus is a much smoother function, with a minimum at 0.21 ps. The inset of Fig. 5 shows the integrals up to time $t$,

$$
\eta(t)=\int_{0}^{t} G(t) d t
$$

for both shear relaxation moduli. [The shear relaxation modulus itself is given by the slope of $\eta(t)$.] It is seen that the atomic and molecular integrals become identical for $t$ larger than $1 \mathrm{ps,}$, in accordance with the findings of other authors. ${ }^{5,11}$ The limit of Eq. (13) for $t$ going to infinity gives the viscosity of the polyethylene melt. The computational demands for these calculations are very large because the stress tensor is a collective property of the system, yielding only one value per time frame (in practice we can use five, as explained in Sec. III). This means that very long runs are needed to obtain enough statistical accuracy. Because of the larger possible integration time step (50 fs), the molecular stress tensor was used to determine $\eta(t)$ for large time scales.

The rate of convergence of the integral of the shear relaxation modulus depends on the relaxation time of the chain. In principle one should integrate up to, say, $2 \tau_{1}$ to reach the plateau value of the integral. This is, however,

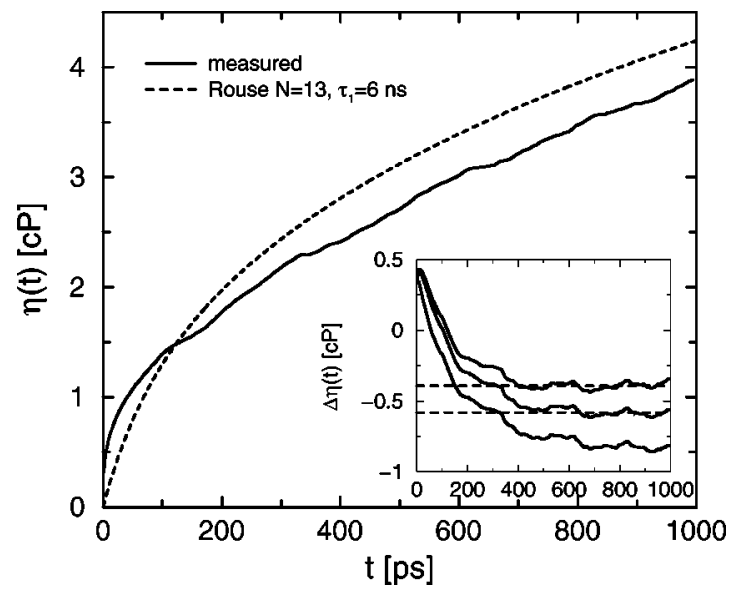

FIG. 6. The long time behavior of the integral of the shear relaxation modulus determined from the molecular stress tensor (line). The dashed line is the Rouse prediction, Eq. (A13), for $N=13$ and $\tau_{1}=6.0 \mathrm{~ns}$. The inset shows the difference $\Delta \eta(t)$ [see Eq. (14)] between measured and Rouse integrals for different sets of parameters. From top to bottom: $\left(N=13, \tau_{1}=6.0 \mathrm{~ns}\right),(N$ $\left.=14, \tau_{1}=6.5 \mathrm{~ns}\right)$, and $\left(N=20, \tau_{1}=7.0 \mathrm{~ns}\right)$. For the first two sets, the difference becomes constant after approximately $0.4 \mathrm{~ns}$ (dashed lines). From this graph we are unable to determine which of the two sets of parameters provides the best fit.

quite impossible, so instead of varying the parameters of the Rouse model ( $N$ and $\tau_{1}$ ) to fit the entire curve, we will use the parameters found before to compare the Rouse predicted integral with the measured integral $\eta(t)$ up to $t=1 \mathrm{~ns}$. The measured result, shown in Fig. 6, is an average of the results of the four independent simulation boxes, each yielding five independent contributions. We can make a conservative estimate of the error in the average value at each time by treating these 20 contributions at each time as independent data. This way we find estimated errors of $6 \%$ at $t=0.1 \mathrm{~ns}$ up to $17 \%$ at $t=1 \mathrm{~ns}$. Because the statistical uncertainties are relatively large, we must be careful with our conclusions.

Before testing the Rouse predictions, let us compare our EMD result with the NEMD results of Moore et al. on $\mathrm{C}_{100}$ chains. $^{8}$ They measured transient shear stresses by applying sudden constant shear rates to equilibrated systems. At the high shear rates applied by these authors, the stresses exhibit temporary overshoots above the steady-state values, in partial agreement with the Doi-Edwards theory of entangled systems. ${ }^{30}$ This seems to indicate that under some conditions aspects of the reptation picture may be applicable, even for chains which are not supposed to be entangled. Notice that our Fig. 6 represents the zero-shear limit of their Fig. 1. In this sense the results of Moore et al. and our results are complementary.

Let us now compare the results with the Rouse integral, Eq. (A13). The density of Rouse chains is set equal to the density of chains in the MD system. The result is shown in Fig. 6 for $N=13$ and $\tau_{1}=6.0 \mathrm{~ns}$. It is immediately apparent that the Rouse curve differs from the measured curve. This should be expected because the initial relaxation modulus $G(t)$ of the polyethylene system does not behave Rouse-like at all, as can be seen in Fig. 5. However, the Rouse curve is observed to run parallel with the measured curve after approximately $0.4 \mathrm{~ns}$. This means that the zero-shear relaxation 
modulus $G(t)$ is perfectly described by the Rouse model in this time regime. Note that $0.4 \mathrm{~ns}$ is the time at which the atoms have moved on average over one statistical segment length $b$. That the two curves really are parallel is best revealed by plotting the difference against time,

$$
\Delta \eta(t)=\eta(t)-\eta^{R}(t)
$$

and observing that $\Delta \eta$ becomes constant after a certain time. The inset of Fig. 6 shows the difference for the three sets of Rouse parameters obtained so far, $N=13$ and $\tau_{1}=6.0 \mathrm{~ns}, N$ $=14$ and $\tau_{1}=6.5 \mathrm{~ns}$, and $N=20$ and $\tau_{1}=7.0 \mathrm{~ns}$. Only the first two sets give a constant $\Delta \eta$ within the measured time span, with a limiting value of $\Delta \eta_{\infty}=-0.39 \mathrm{cP}$ for $N=13$ and $\tau_{1}=6.0 \mathrm{~ns}$, and $\Delta \eta_{\infty}=-0.58 \mathrm{cP}$ for $N=14$ and $\tau_{1}$ $=6.5 \mathrm{~ns}$. Because of the uncertainty in the data, we are unable to determine which of the two sets of Rouse parameters provides the best fit to the data. In the last set, the Rouse parameters are clearly overestimated, resulting in an ever decreasing difference curve. If the zero-shear relaxation modulus can be perfectly described by the Rouse model after $1 \mathrm{~ns}$ as well, we may estimate the viscosity as

$$
\eta=\eta^{R}+\Delta \eta_{\infty}
$$

where $\eta^{R}$ is the limit of Eq. (A13) for $t$ to infinity (8.25 and $8.96 \mathrm{cP}$, respectively, for the first two sets). This yields an estimate for the viscosity of, respectively, $\eta=7.86 \mathrm{cP}$ (first set) and $\eta=8.38 \mathrm{cP}$ (second set) for our melt of $\mathrm{C}_{120} \mathrm{H}_{242}$ chains at $450 \mathrm{~K}$. Pearson et al. ${ }^{17}$ have measured the viscosity of $n$-alkanes and PE as a function of molecular weight at 450 $\mathrm{K}$, although not at the weight of $\mathrm{C}_{120}$ chains. An interpolation of their data yields $\eta_{\text {exp }}=13.5 \mathrm{cP}$. As with other MD simulations at high packing fractions the viscosity is somewhat underestimated, ${ }^{25}$ in our case by $38 \%$ (for the second set). The important result is that we have shown that the shear relaxation modulus $G(t)$ behaves exactly like that of a Rouse chain on time scales between approximately 0.4 and $1.0 \mathrm{~ns}$. A correction $\Delta \eta_{\infty}$ accounts for the fact that initially a real chain does not behave Rouse-like, but it is relatively small for long chains, in our case only 5\% to $6 \%$. Therefore the much applied practice to estimate the viscosity from the longest relaxation time $\tau_{1}$, Eq. (A14), seems to be justified. We would, however, like to place a word of caution: one may not disregard the possibility that $G(t)$ does not behave Rouse-like at times larger than $1 \mathrm{~ns}$. At larger times, contributions from the interactions between different chains may be important, with relaxation times well exceeding the longest intrachain (configurational) relaxation times. A first indication that such processes are important may be found in the transient stress calculations of Moore et al. which suggest that entanglements may be important even in the case of $\mathrm{C}_{100}$ chains. ${ }^{8}$ Additional evidence for this hypothesis will be presented in a forthcoming paper in which we will coarse grain the melt investigated in this paper in order to reach larger time regimes. We will show that on the largest time scales the stress in the system relaxes more slowly than in the case of a Rouse chain, leading to an increased viscosity as compared to the Rouse model.

\section{CONCLUSIONS}

We have performed MD simulations of a polyethylene melt to investigate the validity of the Rouse model predictions for a comprehensive set of correlation functions: the mean square displacement, dynamic structure factor, end-toend vector autocorrelation, and zero-shear relaxation modulus. It was found that the chains do indeed behave like Rouse theory predicts, but only on length scales larger than the segment length $b$. Further, it was found that the different quantities consistently yielded the same set of fit parameters $N, D$, and $\tau_{1}$. The deviations between the Rouse model calculations and the experimental and simulation data of short time mean square displacements and large $q$ dynamic structure factors were shown by Harnau and coworkers to be due to the neglect of molecular stiffness ${ }^{26}$ which becomes prominent on length scales smaller than the statistical segment length. Other effects, such as an extra (internal) friction, may also be of importance on this length scale, as was pointed out by Richter and coworkers. ${ }^{27-29}$

An important part of this work constituted the calculation of the zero-shear relaxation modulus and viscosity of a melt of chains which is supposed to be described by Rouse dynamics. We have found that a Rouse description of the shear relaxation modulus exactly coincides with the simulation results for time scales between 0.4 and 1.0 ns. This allowed us to extrapolate the integral using Rouse theory, yielding a viscosity of $8.38 \mathrm{cP}$, underestimating the experimental value by $38 \%$. The correction due to initial differences between Rouse and simulated shear relaxation modulus is shown to be relatively small. We do not rule out the possibility that at times larger than $1 \mathrm{~ns}$ intermolecular contributions to the stress relaxation modulus, caused by "entanglements," survive, which will substantially enlarge the calculated viscosity.

\section{APPENDIX A: ROUSE MODEL}

In Rouse theory the excluded volume interactions and hydrodynamic interactions are neglected and the polymer is treated as a chain of $N$ segments connected by harmonic springs with spring constant $k=3 k_{B} T / b^{2}$, where $T$ is the temperature, $k_{B}$ is the Boltzmann's constant, and $b$ is the effective bond length (within an infinite chain). The dynamics of the chain is governed by a Langevin equation of motion with a segmental friction coefficient $\zeta$. The resulting set of coupled differential equations can be solved by transforming to normal coordinates,

$$
\mathbf{X}_{k}=\frac{1}{N} \sum_{n=1}^{N} A_{k n} \mathbf{r}_{n} \quad(k=0, \ldots, N-1),
$$

where $A_{k n}$ is defined as

$$
A_{k n}=\cos \left[\frac{k \pi}{N}\left(n-\frac{1}{2}\right)\right] .
$$

In this expression we include a term $1 / 2$ which assures that the Langevin equations are consistent at the boundaries of the chain. ${ }^{31,32}$ The normal coordinates decay exponentially in time, 


$$
\left\langle\mathbf{X}_{k}(t) \cdot \mathbf{X}_{k}(0)\right\rangle /\left\langle X_{k}^{2}\right\rangle=e^{-t / \tau_{k}},
$$

where the relaxation times $\tau_{k}$ are given by

$$
\tau_{k}=\frac{\zeta b^{2}}{3 k_{B} T}\left[4 \sin ^{2}\left(\frac{k \pi}{2 N}\right)\right]^{-1} \quad(k=1, \ldots, N-1) .
$$

If $k$ is very small compared to $N$, Eq. (A4) can be approximated by

$$
\tau_{k} \approx \frac{\zeta N^{2} b^{2}}{3 \pi^{2} k_{B} T} \frac{1}{k^{2}} .
$$

The slowest relaxation time in the chain, $\tau_{1}$, is the so-called Rouse time. Now we present the Rouse results for the various quantities which have been measured in this work. In the derivation of these expressions we will make no further approximations, i.e., we consider a finite number of segments $N$, and we apply the exact expression for the relaxation times, Eq. (A4).

The mean square displacement of the center of mass of the chain is given by the zeroth normal mode $k=0$, and is equal to

$$
g_{\mathrm{cm}}(t)=6 D t,
$$

i.e., it is diffusive at all times, with a diffusion coefficient given by

$$
D=\frac{k_{B} T}{N \zeta} .
$$

By superposing the internal motions of the chain we find the mean square displacement of a typical segment to be equal to

$$
g_{\text {seg }}(t)=6 D t+6 D \sum_{k=1}^{N-1} \tau_{k}\left(1-e^{-t / \tau_{k}}\right) .
$$

In the limit of $N$ going to infinity, $g_{\text {seg }}$ is subdiffusive $\left(\propto t^{1 / 2}\right)$ for small times and diffusive $(\propto t)$ for larger times. The crossover time between these two regimes is dictated by the slowest relaxation time in the chain, $\tau_{1}$.

The dynamic structure factor of a single chain can be calculated by noting that the random displacements of the normal coordinates are Gaussianly distributed. ${ }^{2}$ After some straightforward calculation we find

$$
\begin{aligned}
S(q, t)= & \frac{1}{N} \exp \left\{-q^{2} D t\right\} \sum_{i=1}^{N} \sum_{j=1}^{N} \exp \left\{-\frac{q^{2} b^{2}}{6}|i-j|\right\} \\
& \times \exp \left\{-2 q^{2} D \sum_{k=1}^{N-1} \tau_{k}\left(1-e^{-t / \tau_{k}}\right) A_{k i} A_{k j}\right\} .
\end{aligned}
$$

The average squared end-to-end vector is related to the number of segments,

$$
\left\langle R^{2}\right\rangle=\left\{\begin{array}{l}
N b^{2} \quad(N \text { even }) \\
\left(N-\frac{1}{N}\right) b^{2} \quad(N \text { odd })
\end{array}\right.
$$

This results in standard random walk behavior for $N$ to infinity. The end-to-end vector autocorrelation is given by the sum of odd normal mode decays:

$$
\frac{\langle\mathbf{R}(t) \cdot \mathbf{R}(0)\rangle}{\left\langle R^{2}\right\rangle}=\frac{\sum_{k \text { odd }} \tau_{k} e^{-t / \tau_{k}}}{\sum_{k \text { odd }} \tau_{k}} .
$$

The characteristic times in Eqs. (A8), (A9), and (A11) can be calculated from the Rouse time by using Eq. (A4).

The relaxation of the stress after a step shear is given by the shear relaxation modulus, which is, in the Rouse case, a simple sum of exponential decays,

$$
G(t)=\frac{\rho R T}{M} \sum_{k=1}^{N-1} e^{-2 t / \tau_{k}},
$$

where $\rho$ is the mass density, $M$ is the molecular weight, and $R$ is the gas constant. In analogy with Eq. (13) in the main text we define $\eta^{R}(t)$ as the integral of the Rouse shear relaxation modulus up to time $t$,

$$
\eta^{R}(t)=\frac{\rho R T}{M} \sum_{k=1}^{N-1} \frac{\tau_{k}}{2}\left(1-e^{-2 t / \tau_{k}}\right) .
$$

The limit of this integral for time to infinity is the viscosity. For $N \rightarrow \infty$ this can be approximated as

$$
\eta \approx \frac{\pi^{2}}{12} \frac{\rho R T}{M} \tau_{1} .
$$

So the viscosity of a Rouse chain is found to scale linearly with chain length $N$ for constant segmental friction coefficient. Experimentally a stronger $N$ dependence is observed in the unentangled regime $\left(\propto N^{1.8}\right)$ because the density and, more important, the segmental friction coefficient $\zeta$ increase with increasing $N .^{17}$

${ }^{1}$ W. Paul, G. D. Smith, and D. Y. Yoon, Macromolecules 30, 7772 (1997).

${ }^{2}$ W. Paul, G. D. Smith, D. Y. Yoon, B. Farago, S. Rathgeber, A. Zirkel, L. Willner, and D. Richter, Phys. Rev. Lett. 80, 2346 (1998).

${ }^{3}$ V. Harmandaris, V. G. Mavrantzas, and D. N. Theodorou, Macromolecules 31, 7934 (1998).

${ }^{4}$ D. Brown, J. H. R. Clarke, M. Okuda, and T. Yamazaki, J. Chem. Phys. 104, 2078 (1996).

${ }^{5}$ M. Mondello and G. S. Grest, J. Chem. Phys. 106, 9327 (1997).

${ }^{6}$ M. Mondello, G. S. Grest, E. B. Webb III, and P. Peczak, J. Chem. Phys. 109, 798 (1998).

${ }^{7}$ J. D. Moore, S. T. Cui, H. D. Cochran, and P. T. Cummings, J. NonNewtonian Fluid Mech. 93, 83 (2000).

${ }^{8}$ J. D. Moore, S. T. Cui, H. D. Cochran, and P. T. Cummings, J. NonNewtonian Fluid Mech. 93, 101 (2000).

${ }^{9}$ V. A. Harmandaris, V. G. Mavrantzas, and D. N. Theodorou, Macromolecules 33, 8062 (2000).

${ }^{10}$ P. E. Rouse, J. Chem. Phys. 21, 1273 (1953).

${ }^{11}$ S. T. Cui, P. T. Cummings, and H. D. Cochran, Mol. Phys. 88, 1657 (1996)

${ }^{12}$ H. J. C. Berendsen and W. F. van Gunsteren, GROMOs Reference Manual (University of Groningen, Groningen, 1987).

${ }^{13}$ W. Paul, D. Y. Yoon, and G. D. Smith, J. Chem. Phys. 103, 1702 (1995).

${ }^{14}$ J.-P. Ryckaert and A. Bellemans, Mol. Phys. 44, 68 (1981).

${ }^{15}$ B. Smit, S. Karaborni, and J. I. Siepmann, J. Chem. Phys. 102, 2126 (1995)

${ }^{16}$ H. J. C. Berendsen, J. P. M. Postma, W. F. van Gunsteren, A. Di Nola, and J. R. Haak, J. Chem. Phys. 81, 3684 (1984).

${ }^{17}$ D. S. Pearson, G. Ver Strate, E. von Meerwall, and F. C. Schilling, Macromolecules 20, 1133 (1987).

${ }^{18}$ B. M. Forrest and U. W. Suter, J. Chem. Phys. 102, 7256 (1995).

${ }^{19}$ W. Tschöp, K. Kremer, J. Batoulis, T. Bürger, and O. Hahn, Acta Polym. 49, 61 (1998); 49, 75 (1998).

${ }^{20}$ P. V. K. Pant and D. N. Theodorou, Macromolecules 28, 7224 (1995).

${ }^{21}$ N. F. A. van der Vegt, W. J. Briels, M. Wessling, and H. Strathmann, J. Chem. Phys. 105, 8849 (1996). 
${ }^{22}$ R. D. Olmsted and R. F. Snider, J. Chem. Phys. 65, 3423 (1976).

${ }^{23}$ P. J. Daivis and D. J. Evans, J. Chem. Phys. 100, 541 (1994).

${ }^{24}$ M. P. Allen, Mol. Phys. 52, 705 (1984).

${ }^{25}$ D. K. Dysthe, A. H. Fuchs, and B. Rousseau, J. Chem. Phys. 112, 7581 (2000).

${ }^{26}$ L. Harnau, R. G. Winkler, and P. Reineker, Europhys. Lett. 45, 488 (1999); Phys. Rev. Lett. 82, 2408 (1999).

${ }^{27}$ D. Richter, M. Monkenbusch, J. Allgeier, A. Arbe, J. Colmenero, B. Farago, Y. C. Bae, and R. Faust, J. Chem. Phys. 111, 6107 (1999).
${ }^{28}$ L. Harnau, J. Chem. Phys. 113, 11396 (2000).

${ }^{29}$ D. Richter, M. Monkenbusch, W. Pykhout-Hintzen, A. Arbe, and J. Colmenero, J. Chem. Phys. 113, 11398 (2000).

${ }^{30}$ M. Doi and S. F. Edwards, The Theory of Polymer Dynamics (Clarendon, Oxford, 1986).

${ }^{31}$ W. J. Briels, "Theory of polymer dynamics," lecture notes, Uppsala, 1994 (unpublished). The full text can be downloaded from http:// www.tn.utwente.nl/cdr/PolymeerDictaat/.

${ }^{32}$ A. Kopf, B. Dünweg, and W. Paul, J. Chem. Phys. 107, 6945 (1997). 